\title{
Assessing complexity in hominid dental evolution: fractal analysis of great ape and human molars.
}

\author{
Cano-Fernández, Hugo ${ }^{1,2}$ and Gómez-Robles, Aida $^{3,4,5}$ \\ 1 Universidad Autónoma de Barcelona \\ 2 Institute of Archaeology, University College London \\ 3 Department of Anthropology, University College London \\ 4 Department of Genetics, Evolution and Environment, University College London \\ 5 Department of Life Sciences, Natural History Museum of London \\ * Corresponding author: Hugo Cano-Fernández (tcrnhc3@ucl.ac.uk)
}

\begin{abstract}
Objectives: Molar crenulation is defined as the accessory pattern of grooves that appears on the occlusal surface of many mammalian molars. Although frequently used in the characterization of species, this trait is often assessed qualitatively, which poses unavoidable subjective biases. The objective of this study is to quantitatively test the variability in the expression of molar crenulation in primates and its association with molar size and diet.
\end{abstract}

Methods: The variability in the expression of molar crenulation in hominids (human, chimpanzee, gorilla and orangutan) was assessed with fractal analysis using photographs of first, second and third upper and lower molars. After this, representative values for 29 primate species were used to evaluate the correlation between molar complexity, molar size and diet using a Phylogenetic Generalized Least Squares (PGLS) regression.

Results: Results show that there are statistically significant differences in fractal dimensions across hominid species in all molars, with orangutan molars presenting higher values of occlusal complexity. Our results indicate that there is no significant association between molar complexity and molar size or diet.

Discussion: Our results show higher levels of occlusal complexity in orangutans, thus supporting previously published observations. Our analyses, however, do not indicate a clear association between molar complexity and molar size or diet, pointing to other factors as the major drivers of complexity. To our knowledge, our study is the first one to use fractal analysis to measure occlusal complexity in primates. Our results show that this approach is a rapid and cost-effective way to measure molar complexity.

Key words: complexity, crenulated molars, fractal analysis, hominids, occlusal surface 


\section{Introduction}

Teeth are the most common and best preserved fossil remains and, as such, they are central in palaeontological research. They show highly variable morphologies that are vital for taxonomic identification and phylogenetic reconstruction (Berger et al., 2015; Haile-Selassie, 2001; Senut, Pickford, \& Gommery, 2018; Silcox, 2001). Teeth are commonly associated with diet and macroevolutionary changes in their shape are usually interpreted as adaptations to exploit food resources (Berthaume, Delezene, \& Kupczik, 2018; Boyer, Evans, \& Jernvall, 2010; Ledogar, Winchester, St. Clair, \& Boyer, 2013; Thiery, Guy, \& Lazzari, 2017). Microevolutionary changes in dental traits are usually considered to be highly heritable and reflective of population history (G. R. Scott, Turner, \& others, 1997).

Molar crenulation is sometimes overlooked as a relevant aspect of dental variability. The occlusal surface of many mammalian teeth presents secondary grooves that are collectively known as crenulations or wrinkles (Figure 1.). Therefore, molar crenulation is defined as the presence of secondary well-defined cristids in each molar cusp (Grine, 1981; Swindler \& Ward, 1988). This trait has been described frequently in fossil and living primates (Berger et al., 2015; Haile-Selassie, 2001; Kraus \& Oka, 1967; Ledogar et al., 2013; Pilloud, Maier, Scott, \& Edgar, 2018; Senut et al., 2018; Silcox, 2001), but it is not usually assessed quantitatively. Describing this trait is particularly important because it is common in primate (Beard et al., 2009; Chaimanee et al., 2003; Coster et al., 2013; Godinot, 2014; Kay et al., 2004; Silcox, 2001; Zhang et al., 2014) and human evolution (Berger et al., 2015; Bermúdez de Castro, Rosas, \& Nicolás, 1999; Carbonell, 1965; Frayer, 1973; Martinón-Torres, Bermúdez de Castro, Gómez-Robles, PradoSimón, \& Arsuaga, 2012; Senut et al., 2001; Simpson et al., 2015; Tobias, 1967).

The developmental origin and potential functional significance of molar crenulation are unclear. It has been pointed out that the topology of the outer enamel surface may be determined by the enamel-dentine junction (EDJ) (Kraus \& Oka, 1967). This hypothesis has been recently supported by comparisons of both surfaces with computed tomography, confirming that the topography of the EDJ is directly related to the landscape of the outer enamel surface. The topology of both surfaces differs in some species, which indicates that the configuration of the EDJ is not the only factor determining occlusal complexity on the enamel surface (Skinner et al., 2010). One possibility is that the flow of nutrients by diffusion into ameloblasts is reduced in slightly concave areas of the EDJ, driving to irregular patterns of enamel deposition (Häkkinen et al., 2019).

The functional significance of thick and crenulated enamel has been associated with a hard diet (Kay, 1981; Ledogar et al., 2013; Martin, Olejniczak, \& Maas, 2003; Vogel et al., 2008). This may be the case in orangutans and pitheciines, whose diet includes hard parts of plants such as seeds or unripe fruit. The thick and less crenulated enamel of chimpanzees can be similarly explained by their varied diet (Constantino, Lucas, Lee, \& Lawn, 2009). Apparently, this hypothesis does not explain the presence of thick enamel and molar crenulations in primates that feed mainly on herbaceous vegetation, such as gorillas and bamboo lemurs. However, it must be considered that these species also 
consume barks and culm, which are also hard materials (Constantino et al., 2009; Eronen et al., 2017).

The objective of this study was to measure the complexity of the crenulation pattern in primates using the well-established theoretical and methodological framework of fractal analysis. Within this context, complexity is understood as the amount of information in a biological structure, measured as the number of parts and/or interactions (McShea, 1996, 2005). Consequently, the roughness of the crenulation pattern, which can be estimated using its fractal dimension, can be considered as a good proxy of its complexity. Fractals are objects whose dimension is not a natural number. Therefore, the value of fractal dimension shows how intricate a pattern is and how efficiently it fills the space (Mandelbrot, 1982).

The primary goal of our study was establishing and evaluating fractal analysis as a methodological approach to measure occlusal complexity, testing the hypothesis that there is variability in complexity across hominids and that orangutans show the most complex molars. As stated above, it has been observed that orangutan molars present more wrinkles than those of other hominids (Kraus \& Oka, 1967), a trend that has been confirmed using computed tomography scans in first and second lower molars (Skinner et al., 2010). These observations, however, have not been tested in a comprehensive sample of upper and lower molars using quantitative methods that can be easily expanded to very large sample sizes of primates or other mammals.

The secondary goal of this study was to test two hypotheses regarding the ultimate factors driving complexity in primate molars. Molar complexity can be potentially associated with size as a byproduct of size increase. Therefore, the hypothesis that molar complexity is associated with molar size was evaluated intra- and interspecifically. Because it has been also proposed that thick and crenulated enamel may have evolved as a response to a hard diet in orangutans and pitheciines (Ledogar et al., 2013; Vogel et al., 2008), an association between diet and molar complexity was tested using data on dietary variation in a broader sample of primate species.

\section{Materials and methods}

\section{Materials}

Upper and lower first, second and third molars from humans and non-human primates were photographed in order to assess their occlusal complexity. Non-human primate molars were photographed at the Museum für Naturkunde of Berlin (MNB) and the Natural History Museum of London (NHML). Large samples of Pongo pygmaeus, Gorilla gorilla and Pan troglodytes were obtained, as well as small samples of other primate species to be used in the analysis of the association between molar complexity, molar size and diet (Table 1.). Human molars were photographed at the American Museum of Natural History and at the Laboratory of Physical Anthropology of the University of Granada (Spain). 
Digital photographs of the occlusal surface of the molars were taken to perform complexity analyses. Unworn or minimally worn molars were selected because enamel wrinkles tend to be erased by dental wear. Molars were positioned with the occlusal surface and the cemento-enamel junction parallel to the lens of the camera. To standardize photographing conditions, a photography stand was used. Two light banks were placed at each side of the photography station to minimize shadowing. In most images, one single molar occupied the photograph frame, although for some small primates (such as pitheciines or cercopithecines), multiple molars were registered in the same image. When there were multiple molars in the frame, only the one in the center was used to calculate the fractal dimension. Most photographs were taken with a camera Nikon D80 and a macro lens A/F Micro-Nikon $105 \mathrm{~mm}, \mathrm{f} / 2.8 \mathrm{D}$, or with a camera Canon EOS 800D with a macro lens Sigma 105mm f/2.8 EX DG. A diaphragm opening of $\mathrm{f} / 32$ or $\mathrm{f} / 22$ was used in order to obtain a maximum depth of field and to focus all the occlusal surface (Gómez-Robles \& Polly, 2012). The size of the images ranged from 4.8 to 22.9 megapixels (MP), and pixel size ranged approximately from 3.84 to $10.41 \mu \mathrm{m}$. The number of pixels capturing the occlusal molar surface ranged approximately from $9.48 \cdot 10^{5}$ to $7.00 \cdot 10^{6}$.

\section{Fractal analysis}

\section{Image processing}

The extraction of the crenulation pattern from the digital photographs was carried out using the software ImageJ (Schneider, Rasband, \& Eliceiri, 2012). The crenulation pattern recorded in the images can be influenced by the coloration of molars. For this reason, molars with dark stains whose grooves could not be clearly differentiated were excluded from analyses. Photographs were binarized to black-and-white representations using a manually established threshold to better represent the crenulation pattern. All the black patches that were not attributed to the crenulation pattern were manually removed in this stage. Once the true crenulation pattern was extracted from the images, three representations were obtained. Firstly, a silhouette pattern was extracted, which represents the area of the occlusal surface covered by grooves (Figure 2.B). Secondly, an edges representation was obtained, which shows only the borders of the molar grooves as a line of one-pixel thickness (Figure 2.C). Thirdly, a skeleton pattern was extracted, which shows a simplification of the grooves as single lines with a width of one pixel (Figure 2.D). The aim of these transformations was to find the representation that better reflected the complexity of the occlusal surface, and to minimize the impact of the differences in the width of the grooves. Therefore, the three representations were obtained for each molar and the fractal analysis results were compared. Results of these comparisons (provided in the 'Results' section below) show strong correlations between the three representations. Still, the edges representation was considered the most reliable because it was less influenced by the coloring of the enamel surface, which has an effect on the apparent width of the grooves in binarized images. By reducing the binarized image to the edge of the crenulations, the effect of the width of the grooves on the quantification of the fractal dimension was minimized across images. 


\section{Box counting method}

The chosen methodology to estimate the fractal dimension was the box counting method. The traditional version of this method superimposes a grid and counts the number of cells that are covered by the object whose fractal dimension is being measured. Then it repeats this process with multiple grids with increasing cell sizes. Next, it performs a linear regression between the logarithm of cell size and the logarithm of the number of cells covered. The fractal dimension is equal to the slope of this regression line multiplied by -1 (Bruner, Mantini, Perna, Maffei, \& Manzi, 2005).

The methodology used in this study was the box counting method with a sliding box, which includes three modifications with respect to the traditional box counting approach. Firstly, instead of the number of boxes occupied by the crenulation pattern, it counts the average of pixels per box. Secondly, each time that the grid is superimposed, its position changes. In other words, the grid slides in each iteration, reducing the effect of the arbitrary position of the cells. Thirdly, the fractal dimension (D) is calculated as the slope of the regression line between the natural logarithm of the average number of pixels per box $(\ln \mu F)$ and the natural logarithm of the ratio of box size between image size $(\ln \varepsilon)$. This eliminates the effect of image size in the final result. To empirically confirm that potential biases related to image size and resolution do not impact results, the resolution of seven photographs of orangutan, chimpanzee and gorilla molars was reduced from 22.9 or 9.6MP to 4MP. Images were processed separately, and the correlation between the obtained fractal dimension values was measured.

Fractal analysis was performed with the FracLac plugin of ImageJ (Schneider et al., 2012) which was used with the default settings. This means that the size of the boxes ranged from $5 \%$ to $100 \%$ of the image size. FracLac optimizes automatically the absolute size of boxes to improve the fit of the regression line; therefore the adjusted $R^{2}$ coefficients were high in all the molars, with a minimum value of 0.882 (Figure 2 . in supplementary information). The target number of box sizes was 10 , which reduces considerably the computing time. The grid was slid 5 pixels in both the " $x "$ and the "y" axes in each iteration. Non-parametric Kruskal-Wallis tests with posthoc pairwise comparisons were performed to determine statistically significant differences in fractal dimension within and across hominid species.

\section{Phylogenetic generalized least squares regression (PGLS)}

A Phylogenetic Generalized Least Squares (PGLS) regression was used to test the potential relationship between occlusal complexity, molar size and diet. This method performs a linear regression between two variables taking into consideration the phylogenetic relationships of the species, as it is expected that closely related taxa will show similar values that do not necessarily reflect association between complexity and molar size or diet (Famoso \& Davis, 2016).

PGLS regressions were performed using representative values of fractal dimension for 29 primate species. These values were obtained by averaging the available measures 
for each species. The phylogenetic tree used for this analysis was obtained from the 10kTrees project (Arnold, Matthews, \& Nunn, 2010). As mentioned above, two variables were selected to test for possible correlations with fractal dimension. First, the size of occlusal surface may be expected to influence complexity, because larger molars have more space to develop intricate grooves. Therefore, the occlusal surface was measured on digital photographs using the Analyse particles tool of Image . Because molar size is highly variable across primates, averaged surface areas were logtransformed. Similarly, the molar size might be associated with complexity at an intraspecific level. Therefore, ordinary linear regressions were performed between occlusal surface area and fractal dimension within each hominid species (Homo sapiens, Pan troglodytes, Gorilla gorilla and Pongo pygmaeus) for all the molars. For the sake of consistency with previous analyses, surface areas were also log-transformed.

In addition, it has been suggested that species' diets may be related to occlusal complexity such that diets including harder items will be associated with higher levels of molar crenulation (Ledogar et al., 2013; Vogel et al., 2008). Dietary variation across genera was quantified as the percentage of fruit in diet, which was obtained from a previously published compilation (DeCasien, Williams, \& Higham, 2017). Because fruit is a soft food, a negative correlation between molar complexity (measured as the fractal dimension) and diet (measured as the percentage of fruit) is expected. This database does not include some of the taxa whose fractal dimension has been estimated. Therefore, only 17 species were used in these analyses. All statistical analyses were carried out in R.3.4.0.

\section{Results}

\section{Preliminary analyses}

Our preliminary analyses demonstrated that images with different resolutions can be used together within the same analysis. After processing and analyzing the images separately, a 1:1 relationship with a strong linear correlation (adjusted $R^{2}=0.9681$ and P-value $=2.58 \cdot 10^{-11}$ ) was found between the fractal dimension obtained with a high and low resolution of the same molars. This demonstrates that images with different resolutions can be analyzed together in the conditions tested in our study (Figure 1. in supplementary information).

The initial comparison between the three representation approaches (silhouette, edges and skeleton) showed a strong linear correlation in the fractal dimension values between the three representations $\left(R^{2}=0.816\right.$ and P-value $<2.2 \cdot 10^{-16}$ for skeleton and edges; $R^{2}=0.7526$ and P-value $<2.2 \cdot 10^{-16}$ for silhouette and edges; $R^{2}=0.801$ and P-value $<2.2 \cdot 10^{-16}$ for silhouette and skeleton). The edges representation, however, was considered the best estimate of the complexity of the occlusal surface because it is less influenced by the apparent width of the grooves after binarization of the images. Therefore, we focus our results and discussion below on those obtained with the edges representation. 
Our first hypothesis was that there are differences in the complexity of the crenulation pattern in hominid molars, with orangutans showing the most complex molars across hominids. Figure 3. shows the results of the analysis of the fractal dimension in each molar for the four hominid species (corresponding to the edges representation as explained in the Methods section). As expected, results show statistically significant differences, with orangutans showing a significantly higher fractal dimension than all the other three groups in every molar (Table 1. in supplementary information). In general, complexity values in chimpanzees show a wide range of variation, but they are not significantly different from the rest of species in most of the cases. Gorillas and humans show in general similar results, although the fractal dimension of gorillas was significantly smaller in the upper second molar.

Fractal dimension values were also compared between the three molars within each species. No statistical differences were found between M1, M2 and M3 (upper and lower) for orangutans or gorillas. Conversely, chimpanzees and humans showed some significant differences across molars, although a consistent pattern was not observed. In humans, the upper first molar had a significantly lower fractal dimension than all the lower molars, and the lower second molar was significantly less complex than the lower third molar. In chimpanzees, the upper first molar was significantly less complex than the rest of the upper molars (Table 2. in supplementary information). In general, these results do not show clear differences in levels of complexity across the molar rows of any hominid species, which can initially point to a lack of functional specialization of this trait among teeth.

\section{Size and complexity}

Our second hypothesis was that there is a correlation between size and complexity in primate molars. This correlation may exist within or across species. Refuting our hypothesis, ordinary linear regressions between the logarithm of the occlusal area (in $\mathrm{mm}^{2}$ ) and fractal dimension showed no significant correlation in any of the molars in any of the hominid species.

The correlation between size and complexity may be a general macroevolutionary trend that is not observed intraspecifically. A PGLS regression was performed with 34 different primate species. Table 2 . shows the slope, the multiple $R^{2}$ coefficient and the P-value for the PGLS model in each molar. Most correlations between these variables are not significant but the lower first molar shows a highly significant correlation $\left(R^{2}\right.$ $=0.54$; P-value $<0.001)$ and the lower second molar a moderate but significant correlation $\left(R^{2}=0.20\right.$; P-value $\left.=0.040\right)$. These results show a generally closer association between size and complexity for lower molars than for upper molars, although this relationship is generally weak and not consistent across the complete lower molar row. 


\section{Diet and occlusal complexity}

Our final hypothesis was that there is a correlation between molar occlusal complexity and diet in primates. In order to test this hypothesis, a PGLS regression was performed between the representative values of fractal dimension and the percentage of fruit in diet for 17 species. Table 3. shows the statistical summary of the model in each molar. In this case, there are also positive and negative correlations, although they were in general weak and non-significant. The only border-line significant correlation corresponds to the lower first molar $\left(R^{2}=0.27\right.$; P-value $\left.=0.080\right)$. These results show a general lack of association between diet and molar complexity, at least as far as dietary variation can be quantified by the variables used here.

\section{Discussion}

\section{Quantitative measurements of occlusal complexity}

In this study, the well-established theoretical framework of fractal analysis has been used to measure the complexity of the crenulation pattern in hominid molars. Our results show that there are statistically significant differences between hominid species, with orangutans showing higher levels of molar complexity (Figure 3.). These results confirm previous qualitative observations about the expression of molar crenulation (Kraus \& Oka, 1967), and allow for the establishment of a time- and costeffective approach to quantitatively assess this trait in extant or fossil specimens.

Although fractal analysis provides a straightforward way to measure complexity in large samples of molars without the need of computed tomography scans, this methodology has some limitations. These limitations stem from the fact that photography is a variable process and, therefore, some factors may influence the final results. One such factor is the scale of the images, reflected in differences in pixel size and the number of pixels registering the occlusal surface. The method of box counting with sliding box reduces the effects of scale using the ratio between box size and image size instead of the absolute box size to calculate the fractal dimension. This has been confirmed by our empirical tests, which show that the results of fractal dimension using the described methodology and in the conditions of our study are replicable with different image resolutions (Figure 1. in supplementary information). Other factors that may potentially influence our results are the differences in the coloration of enamel surface and in image brightness. While brightness differences can be minimized through the use of standardized lighting sources, differences in enamel coloration are unavoidable in museum specimens. These differences affect mostly the width of the grooves registered in the images, as other stains on the enamel have been removed manually. The use of the edges representation (i.e., the use of the external contour of the crenulation pattern after binarization and manual correction) minimizes this effect and makes molars from different individuals more comparable.

Assessing molar crenulation quantitatively is important in palaeoanthropology because it is present in the first description of some fossil hominin species, such as Homo naledi 
or Ardipithecus kadabba (Berger et al., 2015; Haile-Selassie, 2001; Simpson et al., 2015). However, previous publications have described the presence or absence of crenulations using qualitative adjectives such as coarse, dense or heavy (Godinot, 2014; Ji et al., 2013; Rasmussen, 1996). In some cases, crenulation patterns have also been compared with reference species. For example, the occlusal surface of Ardipithecus and Orrorin has been compared with chimpanzees (Haile-Selassie, 2001; Senut et al., 2018). However, the results obtained here show that the complexity of the crenulation pattern in the genus Pan is particularly variable, making it difficult to speak about a Pan-specific level of crenulation (Figure 3.). Our results show that the intraspecific variability in this trait should be considered when comparing fossil and living primates.

Other quantitative approaches have been used to assess occlusal complexity. One such methodology, which is based on 3D virtual models of molars typically obtained from a computed tomography scan, is the Orientation Patch Count (OPC). In this approach, the occlusal surface is divided in several patches, after which complexity (OPC) is described as the number of patches with distinct orientation (Evans, Wilson, Fortelius, \& Jernvall, 2007). This method has been used to assess enamel complexity in apes, prosimians (Thiery, Guy, \& Lazzari, 2019), pitheciines (Ledogar et al., 2013), plesiadapiforms (Boyer et al., 2010), australopiths and Homo naledi (Berthaume et al., 2018). In addition, it was also used to compare the topography of the outer occlusal surface with the EDJ in hominids and other primates (Skinner et al., 2010).

These studies aimed to measure the complexity of all the molar occlusal surface, whereas fractal analysis as implemented in our study only measures the complexity of the crenulation pattern. In other words, it indicates how intricate the pattern of grooves is without considering the main cusps, thus excluding variation that can have a stronger functional role. Accordingly, the lack of association observed between molar complexity and diet is not surprising, as dental topography as determined by major cusps is not included in our measurements of molar complexity. Instead, fractal dimension seems to be reflecting variation that may be functionally neutral, even if its ultimate causes remain unclear (see below). In any case, although the results obtained with the fractal dimension are generally consistent with those found with an OPCR approach (Figure 3. in supplementary information), these two techniques may give complementary information (Skinner et al., 2010).

\section{Size and complexity}

Our study has evaluated two potential evolutionary influences on molar complexity. This trait may be linked to molar size, such that larger molars may be more complex than smaller ones. This hypothesis has been already proposed for the teeth of horses (Famoso \& Davis, 2016). Instead of molar crenulation, the occlusal surface of equids has a characteristic pattern of enamel bands. The correlation between surface area and complexity was tested in fossil and extant horses from the tribes Equini and Hipparionini. This study showed a weak and non-significant correlation between molar size and complexity in both tribes (Famoso \& Davis, 2016). 
The potential association between molar size and complexity was tested here by comparing the fractal dimension and occlusal surface for 21 primate species using a PGLS regression. Correlations are generally not significant with the exception of the lower first and second molars (Table 2.). These results confirm to a certain extent the previously discussed research in fossil and living horses. Similarly, the ordinary regressions between fractal dimension and occlusal surface within the four hominid species did not yield any significant correlation. Our results, therefore, do not clearly support a strong association between molar size and complexity, although they do indicate a weak association observed only in lower molars. The general lack of association between molar size and complexity is not surprising because size only gives the potentiality to be complex, but these two properties are not necessarily linked (Edmonds, 1995). For example, the molars of pitheciines are small, but they have a highly intricate crenulation pattern (Figure 1.E).

\section{Diet and occlusal complexity}

The second explanatory factor for molar crenulation explored in this study was diet. Traditionally, it has been accepted that thick and crenulated enamel is associated with a diet based on hard items (Kay, 1981). This hypothesis is supported by the evidence of chimpanzees and orangutans. When ripe fruit is not available, orangutans rely on seeds and other hard resources. As a result, they are thought to have developed a thick and crenulated enamel. Conversely, chimpanzees search for more fruit or they eat herbaceous terrestrial vegetation. Therefore, their enamel is thin, less crenulated and they have higher cusps (Constantino et al., 2009; Vogel et al., 2008). This model can be considered to be supported by the results of our study, as occlusal complexity was significantly higher in Pongo that in Pan. Although the correlation between diet and molar complexity is not clear, this association has been assumed for other fossil species (Candela, Cassini, \& Nasif, 2013; Kay et al., 2004; Martin et al., 2003).

In any case, our explicit evaluation of the association between molar complexity and diet does not support such a relationship. The measurement of dietary variation used in our study was the percentage of fruit, which is a soft and brittle material. We would have expected a negative correlation between this variable and fractal dimension if highly complex teeth are an adaptation to exploit hard resources. PGLS regression analyses yielded non-significant positive correlations in all molars with the exception of the lower first molar (Table 3.). This evidence does not support our hypothesis about a possible relationship between molar complexity and diet. It is possible, however, that molar crenulation is not an adaptation to exploit a primary resource, but it may have evolved as an adaptation to use the secondary resources that are consumed when fruit is scarce (Constantino et al., 2009). Therefore, more detailed dietary data are necessary to test the potential effect of dietary variation on molar complexity properly.

\section{Evolutionary-developmental explanations of molar crenulation}

Taking into consideration recent advances in the study of dental development, a different hypothesis can be tentatively proposed to explain enamel crenulation. It has been shown that the topology of the outer enamel surface, and thus the origin of enamel 
crenulations, is partially determined by the EDJ (Skinner et al., 2010). The whole complexity of the outer enamel surface, however, cannot always be explained by a geometric correlation with the EDJ. Developmental models indicate that nutrients do not arrive homogeneously to all the ameloblasts in the slightly convex areas of the EDJ, creating a pattern of differential enamel deposition. This developmental mechanism is non-linear, so small perturbations in the initial conditions can result in large changes in the final pattern of enamel crenulations in the outer surface (Häkkinen et al., 2019). These non-linear dynamics are well known and have been described in models of dental development (Salazar-Ciudad \& Jernvall, 2005). More critically, the complex relationship between genotype and phenotype sometimes makes it possible to select for general properties but not for specific forms (Salazar-Ciudad \& Marín-Riera, 2013). One of those general properties can be complexity, but molar crenulations per se may be simply a byproduct of dental development with no impact on global fitness and, therefore, lacking a functional explanation. More research is needed to reveal whether crenulations have a significant effect on the physical properties of molars and whether they have an impact on the fitness of primate species.

\section{Conclusions}

Our study set up and evaluated a methodology to measure molar complexity based on the well-known theoretical and methodological framework of fractal analysis. Confirming previous qualitative and quantitative observations, we have demonstrated that fractal analysis based on photographs is a time- and cost-effective method to measure molar complexity across large samples of extant and fossil primates, and probably of other mammals.

Three particular hypotheses were tested using this methodology. Firstly, it was hypothesized that there are differences in the expression of molar crenulation within hominids. Our results showed that there are indeed statistically significant differences in occlusal complexity between the different hominid genera, with orangutans showing the most complex molars, chimpanzees showing intermediate average values but large ranges of variation, and gorillas and humans showing the lowest levels of complexity. These results are generally consistent across all the molars within the upper and lower molar rows.

Our second hypothesis was that molar complexity is related to molar size measured as the area of the occlusal surface. In this case, our results showed that there is not a consistent correlation between molar size and complexity. However, our results also show that lower molars have a slightly closer relationship between size and complexity than upper molars. In any case, our study does not support a correlation between size and complexity as a general explanation for molar crenulation.

Finally, another potential ultimate explanation was tested, namely whether molar complexity is related to diet. In this case, our results showed that there is not a clear association between dietary variation and molar complexity. Our dietary data however, may not reflect the complexity of dietary variation that different primate species are 
exposed to, so more analyses including other dietary measurements would be required to further explore this association.

\section{Data availability statement}

The data that support the findings of this study are available from the corresponding author, H.C.-F., upon reasonable request.

\section{Acknowledgements}

We are grateful to Roberto Portela-Miguez, Frieder Mayer, Miguel Botella and Ian Tattersall for facilitating access to the collections at the Natural History Museum, Museum für Naturkunde, University of Granada, and American Museum of Natural History, respectively. We also want to thank professors José Manuel Cano-Izquierdo, Simon Hillson and Mark Lake for their useful comments. This study was originally carried out as the MSc dissertation project of H.C.-F. within the MSc in Palaeoanthropology and Palaeolithic Archaeology of UCL. A.G.-R. is funded by the UCLExcellence programme.

\section{Bibliography}

Arnold, C., Matthews, L. J., \& Nunn, C. L. (2010). The 10kTrees website: A new online resource for primate phylogeny. Evolutionary Anthropology: Issues, News, and Reviews, 19(3), 114-118.

Beard, K. C., Marivaux, L., Chaimanee, Y., Jaeger, J.-J., Marandat, B., Tafforeau, P., ... Kyaw, A. A. (2009). A new primate from the Eocene Pondaung Formation of Myanmar and the monophyly of Burmese amphipithecids. Proceedings of the Royal Society B: Biological Sciences, 276(1671), 3285-3294.

Berger, L. R., Hawks, J., Ruiter, D. J. de, Churchill, S. E., Schmid, P., Delezene, L. K., ... others. (2015). Homo naledi, a new species of the genus Homo from the Dinaledi Chamber, South Africa. Elife, 4, e09560.

Bermúdez de Castro, J. M., Rosas, A., \& Nicolás, M. E. (1999). Dental remains from Atapuerca-TD6 (Gran Dolina site, Burgos, Spain). Journal of Human Evolution, 3(37), 523-566.

Berthaume, M. A., Delezene, L. K., \& Kupczik, K. (2018). Dental topography and the diet of Homo naledi. Journal of Human Evolution, 118, 14-26.

Boyer, D. M., Evans, A. R., \& Jernvall, J. (2010). Evidence of dietary differentiation among late Paleocene-early Eocene plesiadapids (Mammalia, Primates). American Journal of Physical Anthropology, 142(2), 194-210. 
Bruner, E., Mantini, S., Perna, A., Maffei, C., \& Manzi, G. (2005). Fractal dimension of the middle meningeal vessels: Variation and evolution in Homo erectus, Neanderthals, and modern humans. European Journal of Morphology, 42(4-5), 217-224.

Candela, A. M., Cassini, G. H., \& Nasif, N. L. (2013). Fractal dimension and cheek teeth crown complexity in the giant rodent Eumegamys paranensis. Lethaia, 46(3), 369-377.

Carbonell, V. M. (1965). The teeth of the Neanderthal child from Gibraltar: A reevaluation. American Journal of Physical Anthropology, 23(1), 41-49.

Chaimanee, Y., Jolly, D., Benammi, M., Tafforeau, P., Duzer, D., Moussa, I., \& Jaeger, J.-J. (2003). A Middle Miocene hominoid from Thailand and orangutan origins. Nature, 422(6927), 61-65.

Constantino, P. J., Lucas, P. W., Lee, J. J.-W., \& Lawn, B. R. (2009). The influence of fallback foods on great ape tooth enamel. American Journal of Physical Anthropology, $140(4), 653-660$.

Coster, P., Beard, K. C., Soe, A. N., Sein, C., Chaimanee, Y., Lazzari, V., ... Jaeger, J.-J. (2013). Uniquely derived upper molar morphology of Eocene Amphipithecidae (Primates: Anthropoidea): homology and phylogeny. Journal of Human Evolution, 65(2), 143-155.

DeCasien, A. R., Williams, S. A., \& Higham, J. P. (2017). Primate brain size is predicted by diet but not sociality. Nature Ecology \& Evolution, 1(5), 0112.

Edmonds, B. (1995). What is Complexity?-the philosophy of complexity per se with application to some examples in evolution. In F. Heylighen, J. Bollen, \& A. Riegler (Eds.), The evolution of complexity (pp. 1-18). Dordrecht: Kluwer.

Eronen, J. T., Zohdy, S., Evans, A. R., Tecot, S. R., Wright, P. C., \& Jernvall, J. (2017). Feeding ecology and morphology make a bamboo specialist vulnerable to climate change. Current Biology, 27(21), 3384-3389.

Evans, A. R., Wilson, G. P., Fortelius, M., \& Jernvall, J. (2007). High-level similarity of dentitions in carnivorans and rodents. Nature, 445(7123), 78-81.

Famoso, N. A., \& Davis, E. B. (2016). On the relationship between enamel band complexity and occlusal surface area in Equids (Mammalia, Perissodactyla). PeerJ, 4, e2181.

Frayer, D. W. (1973). Gigantopithecus and its relationship to Australopithecus. American Journal of Physical Anthropology, 39(3), 413-426.

Godinot, M. (2014). Fossil record of the primates from the Paleocene to the Oligocene. In W. Henke \& I. Tattersall (Eds.), Handbook of paleoanthropology: Vol II: Primate evolution and human origins (pp. 1-102). Berlin: Springer. 
Gómez-Robles, A., \& Polly, P. D. (2012). Morphological integration in the hominin dentition: Evolutionary, developmental, and functional factors. Evolution, 66(4), 1024-1043.

Grine, F. E. (1981). Occlusal morphology of the mandibular permanent molars of the South African Negro and the Kalahari San (Bushman). Annals of the South African Museum, 86(5), 157-215.

Haile-Selassie, Y. (2001). Late Miocene hominids from the middle Awash, Ethiopia. Nature, 412(6843), 178-181.

Häkkinen, T. J., Sova, S. S., Corfe, I. J., Tjäderhane, L., Hannukainen, A., \& Jernvall, J. (2019). Modeling enamel matrix secretion in mammalian teeth. PLoS Computational Biology, 15(5), e1007058.

Ji, X., Jablonski, N. G., Su, D. F., Deng, C., Flynn, L. J., You, Y., \& Kelley, J. (2013). Juvenile hominoid cranium from the terminal Miocene of Yunnan, China. Chinese Science Bulletin, 58(31), 3771-3779.

Kay, R. F. (1981). The nut-crackers-a new theory of the adaptations of the Ramapithecinae. American Journal of Physical Anthropology, 55(2), 141-151.

Kay, R. F., Schmitt, D., Vinyard, C. J., Perry, J. M., Shigehara, N., Takai, M., \& Egi, N. (2004). The paleobiology of Amphipithecidae, south Asian late Eocene primates. Journal of Human Evolution, 46(1), 3-25.

Kraus, B. S., \& Oka, S. W. (1967). Wrinkling of molar crowns: New evidence. Science, 157(3786), 328-329.

Ledogar, J. A., Winchester, J. M., St. Clair, E. M., \& Boyer, D. M. (2013). Diet and dental topography in pitheciine seed predators. American Journal of Physical Anthropology, 150(1), 107-121.

Mandelbrot, B. B. (1982). The Fractal Geometry of Nature (1st ed.). New York: W.H. Freeman; Company.

Martin, L. B., Olejniczak, A. J., \& Maas, M. C. (2003). Enamel thickness and microstructure in pitheciin primates, with comments on dietary adaptations of the middle Miocene hominoid Kenyapithecus. Journal of Human Evolution, 45(5), 351-367.

Martinón-Torres, M., Bermúdez de Castro, J. M., Gómez-Robles, A., Prado-Simón, L., \& Arsuaga, J. L. (2012). Morphological description and comparison of the dental remains from Atapuerca-Sima de los Huesos site (Spain). Journal of Human Evolution, 62(1), 758.

McShea, D. W. (1996). Perspective. Metazoan complexity and evolution: Is there a trend? Evolution, 50(2), 477-493.

McShea, D. W. (2005). The evolution of complexity without natural selection, a possible large-scale trend of the fourth kind. Paleobiology, 31(S2), 146-156. 
Pilloud, M., Maier, C., Scott, G., \& Edgar, H. (2018). Molar crenulation trait definition and variation in modern human populations. Homo, 69(3), 77-85.

Rasmussen, T. D. (1996). A new Middle Eocene omomyine primate from the Uinta Basin, Utah. Journal of Human Evolution, 1(31), 75-87.

Salazar-Ciudad, I., \& Jernvall, J. (2005). Graduality and innovation in the evolution of complex phenotypes: Insights from development. Journal of Experimental Zoology Part B: Molecular and Developmental Evolution, 304(6), 619-631.

Salazar-Ciudad, I., \& Marín-Riera, M. (2013). Adaptive dynamics under developmentbased genotype-phenotype maps. Nature, 497(7449), 361-364.

Schneider, C. A., Rasband, W. S., \& Eliceiri, K. W. (2012). NIH Image to ImageJ: 25 years of image analysis. Nature Methods, 9(7), 671-675.

Scott, G. R., Turner, C. G., \& others. (1997). Anthropology of modern human teeth. Cambridge University Press Cambridge.

Senut, B., Pickford, M., \& Gommery, D. (2018). Dental anatomy of the early hominid, Orrorin tugenensis, from the Lukeino Formation, Tugen Hills, Kenya. Revue de Paléobiologie, Genève, 37(2), 577-591.

Senut, B., Pickford, M., Gommery, D., Mein, P., Cheboi, K., \& Coppens, Y. (2001). First hominid from the Miocene (Lukeino formation, Kenya). Comptes Rendus de L'Académie Des Sciences-Series IIA-Earth and Planetary Science, 332(2), 137-144.

Silcox, M. (2001). A Phylogenetic Analysis of Plesiadapiformes and Their Relationship to Euprimates and Other Archontans. Unpublished Ph. D. The Johns Hopkins University.

Simpson, S. W., Kleinsasser, L., Quade, J., Levin, N. E., McIntosh, W. C., Dunbar, N., ... Rogers, M. J. (2015). Late Miocene hominin teeth from the Gona paleoanthropological research project area, Afar, Ethiopia. Journal of Human Evolution, 81, 68-82.

Skinner, M. M., Evans, A., Smith, T., Jernvall, J., Tafforeau, P., Kupczik, K., ... others. (2010). Brief communication: Contributions of enamel-dentine junction shape and enamel deposition to primate molar crown complexity. American Journal of Physical Anthropology, 142(1), 157-163.

Swindler, D. R., \& Ward, S. (1988). Evolutionary and morphological significance of the deflecting wrinkle in the lower molars of the Hominoidea. American Journal of Physical Anthropology, 75(3), 405-411.

Thiery, G., Guy, F., \& Lazzari, V. (2017). Investigating the dental toolkit of primates based on food mechanical properties: Feeding action does matter. American Journal of Primatology, 79(6), e22640.

Thiery, G., Guy, F., \& Lazzari, V. (2019). A comparison of relief estimates used in threedimensional dental topography. American Journal of Physical Anthropology., In press. 
Tobias, P. V. (1967). Olduvai Gorge (1st ed., Vol. 2). Cambridge: Cambridge University Press.

Vogel, E. R., Woerden, J. T. van, Lucas, P. W., Atmoko, S. S. U., Schaik, C. P. van, \& Dominy, N. J. (2008). Functional ecology and evolution of hominoid molar enamel thickness: Pan troglodytes schweinfurthii and Pongo pygmaeus wurmbii.Journal of Human Evolution, 55(1), 60-74.

Zhang, Y., Kono, R. T., Jin, C., Wang, W., Harrison, T., \& others. (2014). Possible change in dental morphology in Gigantopithecus blacki just prior to its extinction: Evidence from the upper premolar enamel-dentine junction. Journal of Human Evolution, 75, 166-171.

\section{Figure legends}

Figure 1. Examples of molar crenulation in living primates. (A) Left LM3 of a chimpanzee (specimen 1939.3383) from the Natural History Museum of London (NHML). (B) Right LM3 of an orangutan (specimen 76.1419) from the NHML. (C) Left LM2 of a gorilla (specimen 1951.9.27.20) from the NHML. (D) Left LM2 of a modern human (specimen LT83(217)) from the Laboratory of Physical Anthropology of the University of Granada. (E) Right LM1, LM2 and LM3 of Cacajao (specimen 28.4.27.4), a pitheciine from the NHML. All the scales show $5 \mathrm{~mm}$.

Figure 2. Right lower second molar of an orangutan from the Museum für Naturkunde of Berlin (specimen 6947). The picture shows the different representations used to assess complexity. (A) Image used to classify the molar with the qualitative scale. The scale bar shows $2 \mathrm{~mm}$. (B) Silhouette representation of the pattern of crenulation. (C) Edges representation. (D) Skeleton representation.

Figure 3. Boxplots showing the differences in fractal dimension between the four studied hominids (human, chimpanzee, gorilla and orangutan). Each graph corresponds to a different molar. The values of fractal dimension were estimated with the box counting method with sliding box. The input pattern was the edges representation. The letters show the statistical significance obtained with the KruskalWallis test with a threshold value of $\mathrm{p}<0.05$. The groups with at least one letter in common are statistically equal and the groups with no letters in common are statistically different. The isolated points show outliers, observations which are placed 1.5 times the interquartile range (IQR) above the upper quartile or below the lower quartile. 
Table 1. Sample size by species for upper and lower molars.

\begin{tabular}{lrrrrrr}
\hline Species & UM1 & UM2 & UM3 & LM1 & LM2 & LM3 \\
\hline Homo sapiens & 14 & 31 & 9 & 14 & 28 & 16 \\
Pan troglodytes & 16 & 25 & 13 & 13 & 20 & 13 \\
Gorilla gorilla & 18 & 23 & 9 & 21 & 23 & 11 \\
Pongo pygmaeus & 23 & 24 & 28 & 33 & 25 & 22 \\
Cacajao calvus & 0 & 0 & 1 & 0 & 1 & 1 \\
Cercocebus torquatus & 5 & 2 & 2 & 0 & 1 & 2 \\
Cercocebus galeritus & 1 & 2 & 1 & 0 & 0 & 0 \\
Cercopithecus cephus & 0 & 1 & 0 & 0 & 1 & 0 \\
Cercopithecus ascanius & 0 & 1 & 1 & 0 & 1 & 1 \\
Cercopithecus nictitans & 0 & 0 & 0 & 0 & 0 & 2 \\
Cercopithecus diana & 1 & 0 & 0 & 0 & 0 & 0 \\
Cercopithecus mitis & 0 & 0 & 0 & 1 & 0 & 0 \\
Colobus polykomos & 4 & 3 & 0 & 1 & 2 & 2 \\
Colobus guereza & 1 & 1 & 1 & 0 & 0 & 0 \\
Hylobates agilis & 1 & 0 & 0 & 0 & 1 & 0 \\
Symphalangus syndactylus & 0 & 1 & 0 & 0 & 0 & 0 \\
Bunopithecus hoolock & 2 & 1 & 0 & 1 & 1 & 0 \\
Hylobates lar & 0 & 1 & 0 & 0 & 0 & 2 \\
Macaca sylvanus & 2 & 2 & 1 & 2 & 0 & 0 \\
Macaca fascicularis & 0 & 1 & 1 & 0 & 1 & 2 \\
Macaca nemestrina & 0 & 0 & 0 & 1 & 1 & 0 \\
Mandrillus leucophaeus & 1 & 6 & 1 & 2 & 3 & 2 \\
Mandrillus sphinx & 2 & 2 & 2 & 0 & 0 & 2 \\
Nasalis larvatus & 4 & 2 & 0 & 1 & 2 & 0 \\
Papio hamadryas & 0 & 3 & 3 & 0 & 0 & 2 \\
Papio ursinus & 2 & 1 & 2 & 0 & 0 & 2 \\
Papio cynocephalus & 2 & 0 & 0 & 1 & 0 & 0 \\
Pithecia pithecia & 0 & 1 & 3 & 0 & 1 & 1 \\
Presbytis melalophos & 2 & 0 & 1 & 2 & 2 & 1 \\
Pygathrix nemaeus & 0 & 2 & 1 & 2 & 2 & 2 \\
Rhinopithecus roxellana & 2 & 3 & 1 & 1 & 4 & 1 \\
Theropithecus gelada & 4 & 3 & 4 & 2 & 4 & 4 \\
Trachypithecus vetulus & 1 & 2 & 0 & 1 & 2 & 1 \\
Trachypithecus johnii & 0 & 0 & 1 & 0 & 0 & 1 \\
\hline \hline & & & & & & \\
\hline
\end{tabular}

1

John Wiley \& Sons, Inc. 
Table 2. Statistical summary for the PGLS regression for occlusal surface area and fractal dimension.

\begin{tabular}{lrrrrrr}
\hline & UM1 & UM2 & UM3 & LM1 & LM2 & LM3 \\
\hline Slope & 0.092885 & 0.049148 & -0.109698 & 0.408857 & 0.210011 & 0.034020 \\
Multiple $R^{2}$ & 0.050482 & 0.015906 & 0.063883 & 0.542497 & 0.204386 & 0.006913 \\
P-value & 0.327495 & 0.557054 & 0.268987 & 0.000746 & 0.039632 & 0.712981 \\
Lambda $(\lambda)$ & 0.926631 & 0.824006 & 0.588880 & 0.000001 & 0.199721 & 0.528198 \\
\hline
\end{tabular}

Number of species compared: UM1 $=21, \mathrm{LM} 1=17, \mathrm{UM} 2=24, \mathrm{LM} 2=21, \mathrm{UM} 3=21, \mathrm{LM} 3=22$. 
Table 3. Statistical summary for the PGLS regression for percentage of fruit in diet and fractal dimension.

\begin{tabular}{lrrrrrr}
\hline & UM1 & UM2 & UM3 & LM1 & LM2 & LM3 \\
\hline Slope & 0.000286 & 0.000564 & -0.000279 & 0.003960 & -0.000003 & -0.000060 \\
Multiple $R^{2}$ & 0.008405 & 0.023510 & 0.003993 & 0.274382 & 0.000000 & 0.000177 \\
P-value & 0.726386 & 0.530854 & 0.809596 & 0.080469 & 0.998775 & 0.959532 \\
Lambda $(\lambda)$ & 0.955845 & 0.724160 & 0.596407 & 0.000001 & 0.687216 & 0.610736 \\
\hline
\end{tabular}

Number of species compared: UM1 $=17, \mathrm{LM} 1=17, \mathrm{UM} 2=12, \mathrm{LM} 2=16, \mathrm{UM} 3=16, \mathrm{LM} 3=17$. 


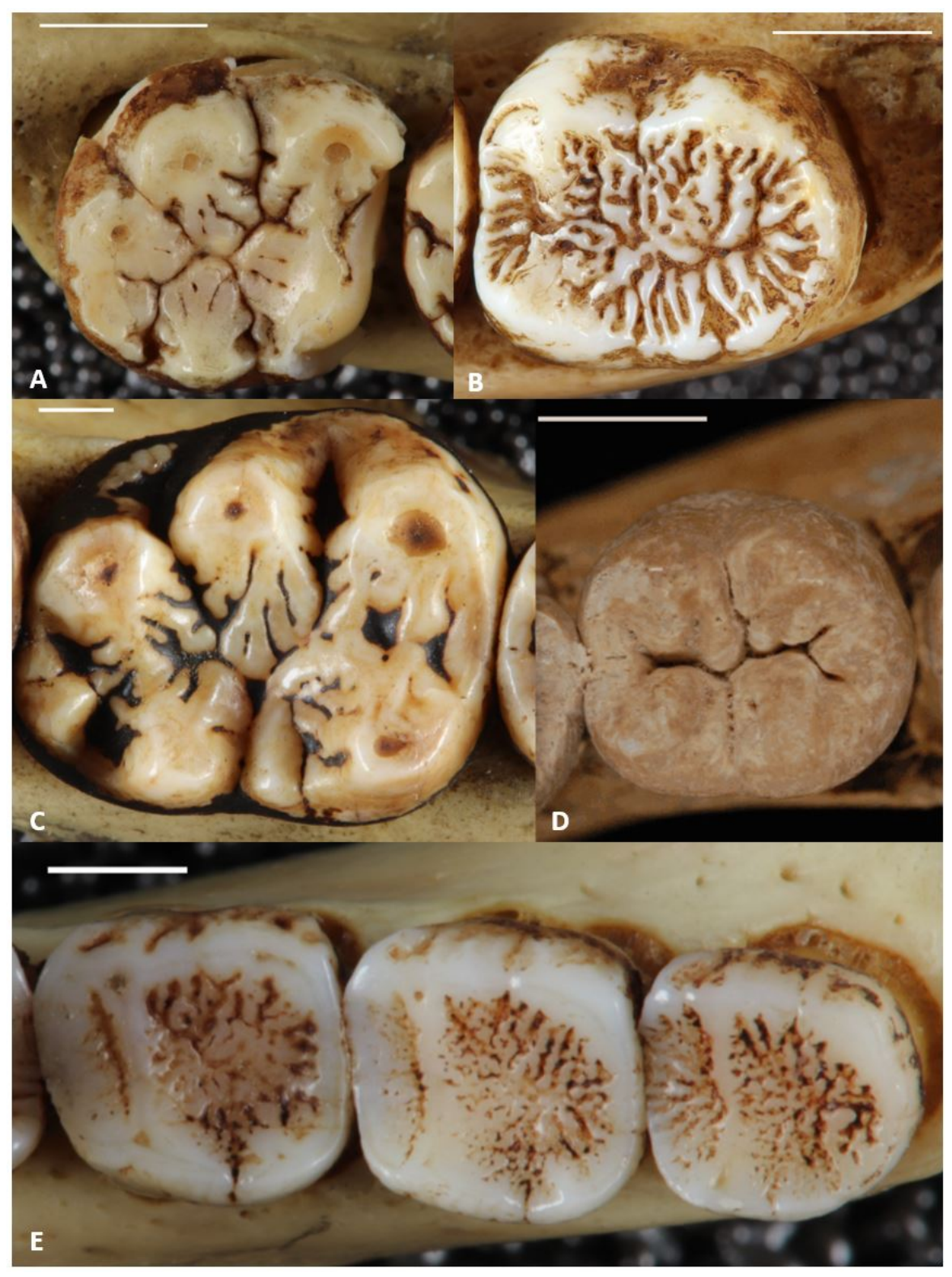

Figure 1. Examples of molar crenulation in living primates. (A) Left LM3 of a chimpanzee (specimen 1939.3383) from the Natural History Museum of London (NHML). (B) Right LM3 of an orangutan (specimen 76.1419) from the NHML. (C) Left LM2 of a gorilla (specimen 1951.9.27.20) from the NHML. (D) Left LM2 of a modern human (specimen LT83(217)) from the Laboratory of Physical Anthropology of the University of Granada. (E) Right LM1, LM2 and LM3 of Cacajao (specimen 28.4.27.4), a pitheciine from the NHML. All the scales show $5 \mathrm{~mm}$.

$73 \times 98 \mathrm{~mm}(300 \times 300$ DPI $)$ 

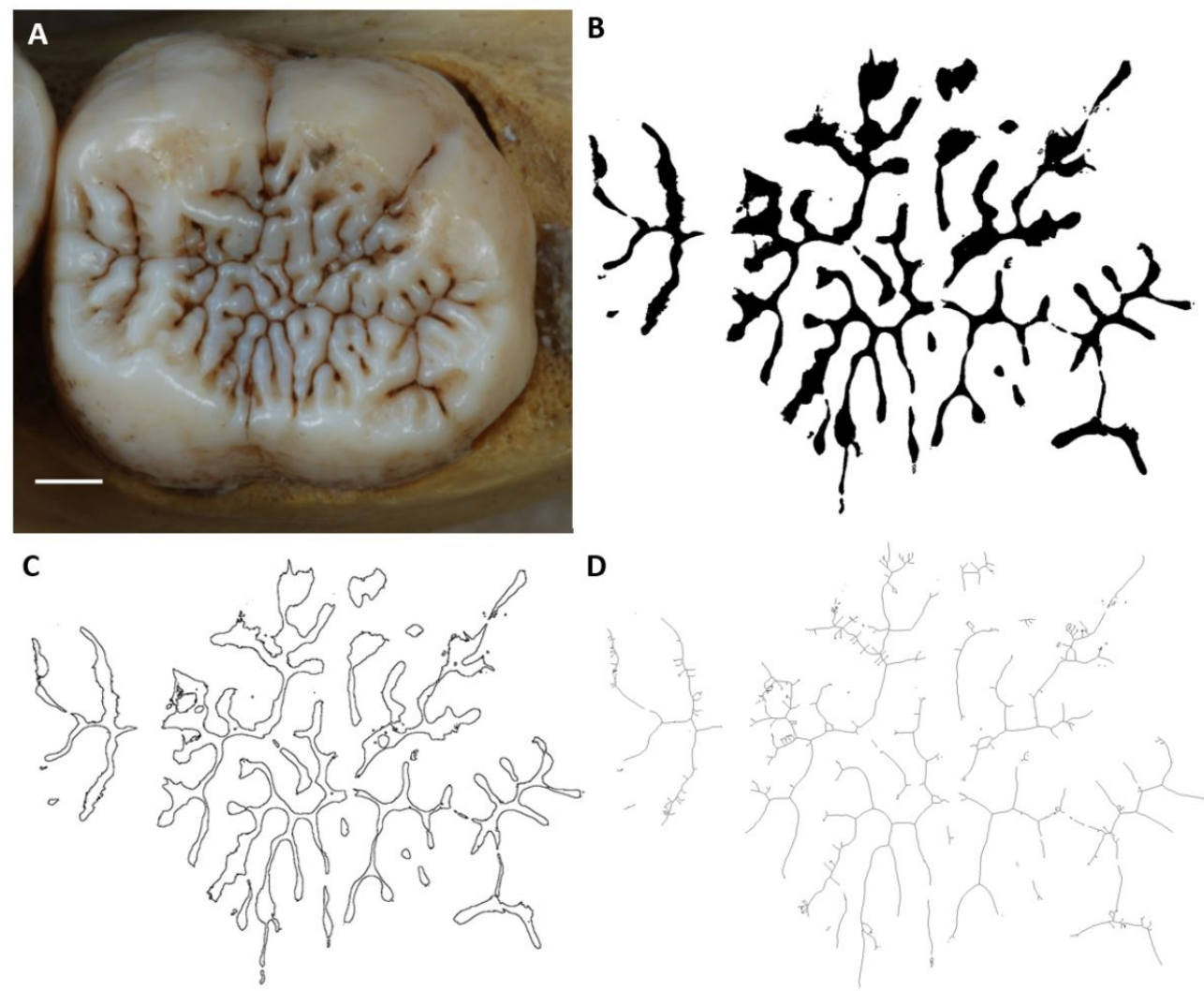

D

Figure 2. Right lower second molar of an orangutan from the Museum fur Naturkunde of Berlin (specimen 6947). The picture shows the different representations used to assess complexity. (A) Image used to classify the molar with the qualitative scale. The scale bar shows $2 \mathrm{~mm}$. (B) Silhouette representation of the pattern of crenulation. (C) Edges representation. (D) Skeleton representation.

$102 \times 84 \mathrm{~mm}(300 \times 300 \mathrm{DPI})$ 

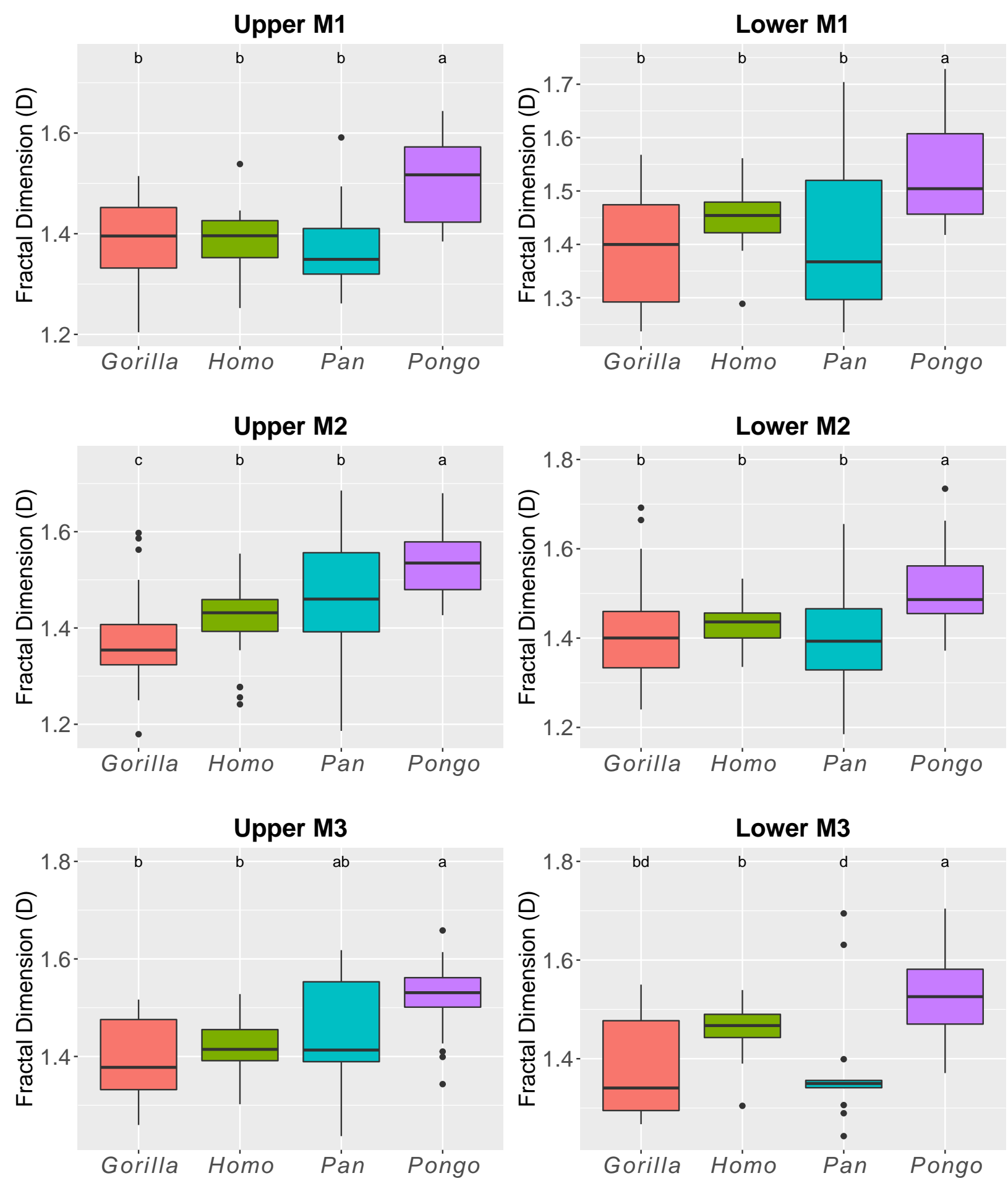